\title{
Hierarchical Production Planning with Part, Spatial and Time Aggregation
}

\author{
J.W. Herrmann \\ Institute for Systems Research, \\ University of Maryland \\ College Park, MD 20742 \\ jwh2@eng.umd.edu
}

\author{
A. Mehra \\ I. Minis \\ Department of Mechanical Engineering and \\ Institute for Systems Research, \\ University of Maryland \\ College Park, MD 20742
}

anshu@eng.umd.edu, minis@eng.umd.edu

\author{
J.M. Proth \\ INRIA-Lorraine, \\ 4 Rue Marconi, Metz 2000 \\ 57070 Metz, France \\ proth@ilm.loria.fr
}

\begin{abstract}
A hierarchical approach to planning production in complex manufacturing systems is presented. A single facility containing a number of work-centers that produce multiple part types is considered. The planning horizon includes a sequence of time periods, and the demand for all part types is assumed to be known. The production planning problem consists of minimizing the holding costs for the work-in-process and finished goods inventory and the backlogging costs for unfulfilled demand. We present a two-level hierurchy that is based on aggregating part types to part families, work-centers to manufacturing cells and time periods to aggregate time periods. The solution of the aggregate optimization problem is imposed as a target in the optimization problems at the detailed level. This architecture uses a rolling horizon strategy to manage production.
\end{abstract}

\section{Introduction}

Production planning determines the quantities of products to manufacture in a sequence of time periods in order to optimize a certain criterion while satisfying constraints such as capacity of resources. This problem is further complicated by random events which may be endogenous (e.g. resource failures) or exogenous (e.g. delayed recejpts of raw material and unexpected changes in demand). The resulting optimization problem is extremely large and complex. Two distinct approaches to production planning have been adopted in the literature: (i) the monolithic approach, wherein the entire problem is formulated as a large mixed-integer/linear programming-type problem, and (ii) the hierarchical approach, which parti- tions the global problem into a series of sub-problems that correspond to different hierarchical levels. These subproblems are solved sequentially and the solution at each level imposes constraints on the solution of the subsequent lower level. While it is impossible to construct a Hierarchical Production Planning (HPP) model which will yield the optimal solution for a given manufacturing system, the major advantages of such an approach are the reduction of complexity and gradual absorption of random events.

Pioneering work on HPP models has been performed by Hax and Meal [1] and extended by others (see references in [4]). The issue of resource aggregation is addressed by few of them and aggregation along time periods is not addressed. We propose a two-level hierarchy which is based on aggregating part types to part families, work-centers to manufacturing cells and time periods to aggregate time periods. The formulation of optimization problems at the aggregate and detailed levels of the proposed hierarchy are presented in this paper. The solution at the aggregate level is imposed as a target in the detailed level planning problems. This architecture uses a rolling horizon strategy to perform the production planning. We present numerical results to demonstrate the effectiveness of the proposed hierarchical approach. In Mehra et al. [3] we address the same planning problem, but the problems at the detailed level are formulated for each cell separately, and a perturbation analysis procedure is employed.

\section{Hierarchical production planning}

In this section, we discuss the production planning problem and the proposed hierarchical approach, which is based on a triple aggregation scheme: (i) part 
types are grouped into part families, (ii) work-centers are grouped into manufacturing cells, and (iii) elementary periods are aggregated into sub-periods.

The two levels in the proposed hierarchical structure are (i) the aggregate (or high) level that plans production for part families on cells within sub-periods and (ii) the detailed (or low) level that disaggregates the high level solution to determine the production plan for part types on work-centers during elementary periods. The criterion at the high level is to minimize inventory costs related to inter-cell work-inprocess (WIP) and finished part families, and backlogging costs of finished part families. At the low level, the criterion is to minimize the holding cost of WIP and finished part types and the backlogging costs of finished part types. Thus, we introduce a hierarchy among inventories; priority is given to inter-cell and end-product inventories. The disaggregation is perforrned over a short-term horizon, which is shorter than the planning horizon. Production planning is usually performed on a rolling horizon basis. That is, although the high level solution is computed over the planning horizon, only a part of it over the short-term horizon is implemented. At the end of the short-term horizon, the plan is recomputed based on the actual state of the system. This is done in order to incorporate the future demands/forecasts progressively.

In Section 2.1 we present the monolithic production planning problem formulation. The hierarchical production planning approach is discussed in Sections 2.2 and 2.3. In Section 2.4 we address the consistency issues associated with this approach. In Section 3, numerical results are presented, and conclusions are presented in section 4 .

\subsection{Monolithic problem formulation}

We consider a single manufacturing facility consisting of a number of manufacturing resources or workcenters. A number of part types are manufactured in the facility. Each part type is produced following a certain routing (a sequence of operations). Each operation is performed on a particular work-center. The processing time of each operation is given; set-up tirnes are ignored in this treatment.

We limit ourselves to a two-level hierarchy in order to simply the proposed hierarchical approach. In this paper we assume that the temporal aggregation has been defined a priori. Furthermore, the aggregation of part types to part families and machines to cells satisfy the following: (A1) Parts belonging to the same part family follow a common sequence of cells during their manufacture. It is not necessary for the parts to follow the same machine sequence. (A2) Parts be- longing to the same part family have similar processing times within each cell. This requirement will result in homogeneity of flows.

We consider a planning horizon $H$ consisting of $Z$ sub-periods (aggregate time periods). Each sub-period is divided into $z$ elementary periods (detailed time periods) of duration $T$ each. Hence, $H=\{1, \ldots, Z$. $z\}$. Let $h(\kappa)=\{(\kappa-1) \cdot z+1, \ldots, \kappa \cdot z\}$ represent the short-term planning horizon associated with subperiod $\kappa$ and $H A$ represent the aggregate planning period $\{1, \ldots, Z\} . T$ is assumed to be much larger than the maximal amount of time needed to perform one operation. Furthermore, $z \cdot T$, which is the duration of a sub-period, is much larger than the time needed to complete the set of operations performed on any part type in any cell. We also assume that: (A3) at most one operation is performed on a part during one elementary period, (A4) a part visits at most one cell during each sub-period, and (A5) a part visits a machine at most once during its visit to a cell.

The production planning problem consists of determining the number of units processed at each operation in a part type's routing during each elementary period of the planning horizon in order to minimize the total holding and backlogging costs. The notation employed in this problem is as follows: (i) $\mathcal{P}$ is the set of $N$ part types; part-types are represented by $j$, (ii) $\mathcal{M}$ is the set of $M$ work-centers; work-centers are represented by $i$, (iii) $\mathcal{R}_{j}$ is the manufacturing routing, i.e. the sequence of operations that part type $j$ must undergo. The total number of operations required for part type $j$ is denoted by $n_{j}$, (iv) $I_{j, w}$ is the holding cost of one unit of part type $j$ for one elementary period after its $w$-th operation is completed, $w \in \mathcal{R}_{j}$, (v) $B_{j}$ is the backlogging cost of one unit of finished part type $j$ for one elementary period, (vi) $t_{j, w}$ is the processing time of the $w$-th operation of part type $j, w \in \mathcal{R}_{j}$, (vii) $\delta_{j, w}(i)$ is the indicator function; assumes a value of 1 if machine $i$ is required for the $w$-th operation of part type $j$, and 0 otherwise, $w \in \mathcal{R}_{j}$, (viii) $d_{j}^{k}$ is the external demand of part type $j$ at the beginning of the $k$-th elementary period, and which must be satisfied by the end of that period, $k \in H$, (ix) $s_{j, 0}^{k}$ is the raw-material inventory for part type $j$ at the end of $k$-th elementary period, $k \in H$, (x) $s_{j, w}^{0}$ is the initial inventory of part type $j$ at the end of $w$-th operation (at the beginning of the first elernentary period), $w \in \mathcal{R}_{j}$, (xi) $s_{j, w}^{k}$ is the inventory of part type $j$ at the end of $k$-th elementary period and at the end of $w$-th operation after satisfying the demand; negative values indicate backlog, $w \in \mathcal{R}_{j}, k \in H$, (xii) $u_{j, w}^{k}$ is the production volume of part type $j$ related to the $w$-th operation on $j$, during the $k$-th elementary 
period, $w \in \mathcal{R}_{j}, k \in H$.

The production planning problem can be described by the optimization problem presented in the Appendix. Although the optimization problem is simple to formulate, there are several reasons why it cannot be solved easily or implemented in practice: (i) the LP is of a very large dimension for a typical manufacturing system and planning horizon, (ii) detailed information about the demand of part types is not known for the entire horizon, (iii) the demand is subject to change due to order cancellations and acceptance of new orders, which requires recomputation, (iv) this formulation does not allow random events to be absorbed with a computation effort proportional to the impact of the random event, and (v) this formulation does not allow different criteria to be used at different levels of hierarchy.

The hierarchical approach to the production planning problem overcomes these problems. In addition, the hierarchical structure parallels the corporate management hierarchy and thus provides significant assistance to the overall management function. The following paragraphs outline the hierarchical approach.

\subsection{Aggregate level problem}

The first step in the proposed hierarchical approach is to solve the aggregate production planning problem This problem concerns the production of part families on manufacturing cells during sub-periods of the planning horizon $(H)$. Notation related to the aggregate level is as follows: (i) $\mathcal{F}$ is the set of $\bar{N}$ part families; note that $\bar{N} \leq N$; a part family is represented by the set $f$, (ii) $\mathcal{C}$ is the set of $\bar{M}$ manufacturing cells; note that $\vec{M} \leq M$; a manufacturing cell is represented by the set $v$, (iii) $\widehat{\mathcal{R}}_{f}$ is the macro-manufacturing process, i.e. the sequence of macro-operations a part family $f$ must undergo. The total number of macro-operations for a part family $f$ are represented by $\bar{n}_{f}$, (iv) $\tilde{I}_{f, q}$ is the inventory cost of one part unit in the representative part family $f$ after the $q$-th macro-operation of $f$ is completed, $q \in \widehat{\mathcal{R}}_{f}$, (v) $\bar{B}_{f}$ is the backlogging cost of one part unit in the representative part family $f$ at the final production stage, $q \in \widehat{\mathcal{R}}_{f}$, (vi) $\tau_{f, q}^{\kappa}$ is the processing time related to the $q$-th macro-operation of one unit of part type in the representative part family $f$ during the $\kappa$-th sub-period, $q \in \widehat{\mathcal{R}}_{f}, \kappa \in H A$, (vii) $D_{f}^{\kappa}$ is the demand of part types in part family $f$ at the beginning of the $\kappa$-th sub-period and is given from

$$
D_{f}^{\kappa}=\sum_{k \in h(\kappa)} \sum_{j \in f} d_{j}^{k}
$$

(viii) $\bar{u}_{f, q}^{\kappa}$ is the production volume of parts in part family $f$ related to the $q$-th macro-operation during; the $\kappa$-th sub-period, $q \in \widehat{\mathcal{R}}_{f}, \kappa \in H A$.

The macro-manufacturing processes (macroroutings) are constructed by aggregating work-centers into cells according to the aggregation rules discussed earlier. For each part type $j \in f$, the routing $\mathcal{R}_{j}$ is partitioned into $\bar{n}_{f}$ sub-routings $\mathcal{R}_{j}^{q}$, each sub-routing corresponding to the $q$-th macro-operation of $f$. Let $v_{j}^{q}$ be the last element in the set $\mathcal{R}_{j}^{q}$.

The computation of inventory/backlogging costs and processing times for families can be obtained from Eqn. (1) - (3). In fact, these parameters depend on the production volume of the part type $u_{j, w}^{k}$. However, since the production volumes are known only after solving the detailed level problem, we use the weighted average of the costs with respect to the part type demands, i.e.,

$$
\begin{gathered}
\bar{I}_{f, q}=\left(\sum_{k \in H} \sum_{j \in f} d_{j}^{k}\right)^{-1} \cdot \sum_{k \in H} \sum_{j \in f} d_{j}^{k} \cdot I_{j, v_{j}^{q}} \\
\vec{B}_{f}=\left(\sum_{k \in H} \sum_{j \in f} d_{j}^{k}\right)^{-1} \cdot \sum_{k \in H} \sum_{j \in f} d_{j}^{k} \cdot B_{j}
\end{gathered}
$$

and the value of $\tau_{f, q}^{\kappa}$ is computed as follows:

$$
\tau_{f, q}^{\kappa}=\frac{z}{z+1-\max _{j \in f} \mid \mathcal{R}_{j}^{q} \|} \cdot \max _{j \in f, u \in \mathcal{R}_{j}^{q}} t_{j, w} .
$$

The symbol | | | represents cardinality. The aggregate level problem can be formally stated as a linear program similar to the monolithic problem. The problem consists of determining the production $\bar{u}_{f, q}^{\kappa}$ over the entire planning horizon for all families at each macro-operation. The production parameters at this level (inventory/backlogging cost, and processing time) correspond to macro-operations of families over sub-periods. The inventory at the end of each macrooperation is estimated at the end of each sub-period for all part families $f \in \mathcal{F}$. The capacity constraints of the aggregate level problem are for each cell in each sub-period of the planning horizon. The aggregate level problem is presented in [2].

\subsection{Detailed level problems}

The detailed level of the hierarchical approach consists of determining the production plan for part types on work-centers during elementary periods of the first sub-period. Recall that we disaggregate only the first sub-period in our rolling horizon approach in order to determine the production volumes $u_{j, w}^{k}$ which respect to the aggregate high level solution $\bar{u}_{f, q}^{\kappa}$ (with $\kappa=1$ ). 
The criterion is to minimize the inventory holding and backlogging costs over the first sub-period.

The disaggregation of the aggregate production volumes $\bar{u}_{f, q}^{k}$ to detailed production volumes $u_{j, w}^{k}$ consist of two steps, the part family disaggregation (PFD) step and the temporal and spatial disaggregation (TSD) step. These two steps are as follows.

\section{Part Family Disaggregation}

At the PFD step, we solve a set of linear programming problems to determine the value of the real variables $\beta_{j, q}^{\kappa} \in[0,1] ; \forall j \in f \in \mathcal{F}, q \in \widehat{\mathcal{R}}_{f}, \kappa \in H A$. These fractions $\beta_{j, q}^{\kappa}$ distribute the production volume of each part family in each macro-operation and sub-period among the part types belonging to that part family. In order to calculate the best distribution of the part family, the disaggregation is done over the entire time horizon $H A$. The variables $\beta_{j, q}^{\kappa}$ satisfy the following equations:

$$
\sum_{j \in f} \beta_{j, q}^{\kappa}=1 ; \forall f \in \mathcal{F}, \kappa \in H A, q \in \widehat{\mathcal{R}}_{f} .
$$

The values of the elements of $\vec{\beta}$ (the vector of variables $\beta_{j, q}^{\kappa}$ ) are obtained from the linear programs presented below. The linear program for each part family is solved independently. Each $\beta_{j, q}^{\kappa}$ is the ratio of expected production in sub-period $\kappa$ of a part type $j$ to the aggregate production volume $\bar{u}_{f, q}^{\kappa}$ obtained from the aggregate optimization problem, i.e.

For each part family $f$, where $f \in \mathcal{F}$, the disaggregation problem at step 1 can be formally stated by the following linear program:

$($ Problem $\mathcal{P} \mathcal{F} \mathcal{D}(f)$ )

$$
\begin{aligned}
& \operatorname{minimize} \sum_{\kappa \in H} \sum_{j \in f}\left\{\sum _ { q = 1 } ^ { n _ { f } - 1 } I _ { j , v _ { j } ^ { q } } \left(s_{j_{, v_{j}^{q}}}^{0}+\sum_{a=1}^{\kappa}\left(\beta_{j, q}^{a} \cdot \bar{u}_{f, q}^{a}-\right.\right.\right. \\
& \left.\left.\beta_{j, q+1}^{a} \cdot \bar{u}_{f, q+1}^{a}\right)\right)+I_{j, n_{j}}\left[s_{j, n_{j}}^{0}+\sum_{a=1}^{\kappa}\left(\beta_{j, \bar{n}_{f}}^{a} \cdot \bar{u}_{f, \bar{n}_{f}}^{a}-D_{f}^{\kappa}\right)\right]^{+} \\
& \left.\quad+B_{j}\left[-s_{j, n_{j}}-\sum_{a=1}^{\kappa}\left(\beta_{j, \bar{n}_{f}}^{a} \cdot \bar{u}_{f, n_{f}}^{a}-D_{f}^{\kappa}\right)\right]^{+}\right\} \\
& \text {subject to: }
\end{aligned}
$$

$$
\text { constraint (4) and }
$$

$$
\beta_{j, q}^{\kappa} \cdot \bar{u}_{f, q}^{\kappa} \leq s_{j, v_{j}^{q-1}}^{0}+\sum_{a=1}^{\kappa}\left(\beta_{j, q-1}^{a} \cdot \bar{u}_{f, q-1}^{a}-\beta_{j, q}^{a} \cdot \bar{u}_{f, q}^{a}\right) ;
$$

$\forall j \in f, q \in \widehat{\mathcal{R}}_{f}, \kappa \in H A$

$$
\beta_{j, q}^{\kappa} \geq 0 ; \forall j \in f, q \in \widehat{R}_{f}, \kappa \in H A .
$$

\section{Temporal and Spatial Disaggregation}

The second step (TSD step) of the disaggregation determines the production of each part type on each operation during each elementary period of the first sub-period $(\kappa=1)$. The production plan at the disaggregation step satisfies the targeted plan obtained from the aggregate level. The production volume of a part family is distributed among the part types according to fractions $\beta_{j, q}^{\kappa}$.

The problem can be formulated as a linear program similar to the monolithic problem:

$$
\begin{aligned}
& \underset{\text { minimize }}{(\text { Problem } \mathcal{T S D}(\kappa))} \sum_{k \in h(\kappa)} \sum_{j \in \mathcal{P}}\left\{\sum _ { w = 1 } ^ { n _ { j } - 1 } I _ { j , w } \left(s_{j, w}^{(\kappa-1) z}+\right.\right. \\
& \left.\left.\sum_{a=(\kappa-1) z+1}^{k}\left(u_{j, w}^{a}-u_{j, w+1}^{a}\right)\right)+P_{j, n_{j}}^{k}\right\} \\
& \text { subject to: } \\
& \text { constraints (11) }-(1.3) ; \quad \forall j \in \mathcal{P} \\
& \text { constraints (11) }-(14) ; \quad \forall k \in h(\kappa) \\
& \sum_{\kappa \in h(\kappa)} u_{j_{, v} q}^{k}=\beta_{j, q}^{\kappa} \cdot \bar{u}_{f, q}^{\kappa} ; \forall j \in f, f \in \mathcal{F}, q \in \widehat{\mathcal{R}}_{f} \\
& \sum_{j \in \mathcal{P}} \sum_{w \in \mathcal{R}_{j}} \delta_{j, w}(i) \cdot u_{j, w}^{k} \cdot t_{j, w} \leq T_{i} \forall i \in M, k \in h(\kappa) \\
& u_{j, w}^{k} \geq 0 ; \forall j \in \mathcal{P}, w \in \mathcal{R}_{j}, k \in h(\kappa) .
\end{aligned}
$$

Constraint (7) defines the production of part type $j$ on the last operation of sub-routing $\mathcal{R}_{j}^{q}$ during the elementary periods of the $\kappa$-th sub-period. This value is determined from the solution of the aggregate level problem and the part family disaggregation problem.

\subsection{Consistency}

Consistency between different levels is ensured in hierarchical approach by the top-down constraints. In hierarchical decisions systems, the term consistency implies that the solution obtained by solving the aggregate optimization problem will yield a feasible solution to the detailed problem. Inconsistencies due to the inappropriate estimation of aggregation parameters may lead to infeasible disaggregation. The consistency for the hierarchical approach presented in this paper is guaranteed in Theorem 1 (proved in [2]).

Theorem 1

There exists at least one feasible solution to problem $\mathcal{T} \mathcal{S} \mathcal{D}(\kappa) \forall \kappa \in H A$, if there exist a feasible solution to the aggregate optimization problem with $\tau_{f, q}^{\kappa}$ defined as in (3). 


\section{Numerical Results}

In this section, we compare the monolithic approach to the hierarchical approach. The parameters compared are the CPU times required and the objective function value obtained. The values of the problem parameters selected (with respect to the problem size parameter $x$ ) for 8 problem sets are presented in Table 1. The second column of Table 1 shows that the relative ratio of these parameters for each problem instance was the same. For all of the problem sets: (i) the maximum number of operations (macrooperations) on any part type (family) is assumed to be the total number of machines (cells) for that problem instance, (ii) the number of elementary periods in a sub-period is 4 , (iii) the number of machines in a cell is 2, (iv) the number of part types in a part family is 4 , and (v) the routing, the holding and backlogging costs, and the processing times of each part type belonging to a part farnily are identical.

Table 1

Parameters for Problem Sets

\begin{tabular}{lc}
\hline \hline Parameter & value \\
\hline \hline Number of Families & $x$ \\
Number of Part types & $4 x$ \\
Number of Cells & $x$ \\
Number of Machines & $2 x$ \\
Number of Sub-periods in $H$ & $4 x$ \\
Number of Elementary Periods in $H$ & $16 x$ \\
\hline$x=$ problem size parameter & \\
$x=1,2, \ldots, 8$ for 8 problem sets &
\end{tabular}

Overall, 40 sample problems were generated, 5 for each problem set. The production planning problems were solved by both the hierarchical and the monolithic approaches. The numerical results were obtained by solving the problems on a SUN/SPARC station. The algorithms were coded in $\mathrm{C}$ and the lin ear programs were solved using XMP. The planning problems were solved on a rolling horizon basis.

Let $C_{h}$ and $C_{m}$ be the objective function values obtained from the hierarchical and monolithic ap. proaches, respectively. Let the CPU times needed by the program in the hierarchical and monolithic ap. proaches be $T_{h}$ and $T_{m}$ respectively. The relative values of these numbers are presented in Table 2. The results show that the hierarchical approach is computationally faster than the monolithic approach even for smaller problems. For larger problems, the hierarchical approach requires very srnall computational effort compared to the monolithi: approach. How ever, the sub-optimality of the planning problem us- ing the hierarchical approach increases as problem size increases. For the sample problems with more than 6 part families, the algorithm for the monolithic approach was terminated due to computer memory constraints. Hence, we have not provided comparison for those sample problems in Table 2. Note that the CPU time needed to solve the problems in set 6 following the monolithic approach was found to be greater than 24 hours.

Table 2

Results

\begin{tabular}{lcc}
\hline \hline $\begin{array}{l}\text { Problem } \\
\text { Set }(x)\end{array}$ & $\begin{array}{c}\text { Mean Value of } \\
\frac{C_{\mathrm{m}}}{C_{h}} \cdot 100\end{array}$ & $\begin{array}{c}\text { Mean Value of } \\
\frac{r_{h}}{T_{\mathrm{m}}} \cdot 100\end{array}$ \\
\hline \hline 1 & $94 \%$ & $24 \%$ \\
2 & $88 \%$ & $1.92 \%$ \\
3 & $86 \%$ & $.21 \%$ \\
4 & $81 \%$ & $.02 \%$ \\
5 & $78 \%$ & $<0.01 \%$ \\
6 & $76 \%$ & $<0.01 \%$ \\
\hline
\end{tabular}

Additional experiments (not reported here) show that the performance of the hierarchical approach depends upon numerous other parameters, including (i) the "similarity" in the holding/backlogging costs, processing times and the routing of part types that belong to the same part family, (ii) the length of the planning horizon, (iii)the number of elementary periods in a sub-period, (iv) the number of part types in a part family, ( $v$ ) the number of work-centers in a cell, and (vi) the capacity utilization. A more comprehensive numerical test is planned to demonstrate this sensitivity. The performance is also affected by the estimation of the part family processing times. One could attack this problem by utilizing an iterative algorithm in which the aggregate processing times are recomputed in order to derive better approximations.

\section{Conclusions}

In this paper, we have presented a general hierarchical approach for solving production planning problems. This approach can be easily extended for other sets of criteria or constraints. Aggregation of part types, machines, and time-periods is considered. A two-level hierarchy is used. At the aggregate level, the production planning of part families on manufacturing cells over aggregate time periods is performed. At the detailed level, the production planning of part types on work-centers over the elementary time periods of the first aggregate time period is performed. A rolling horizon strategy is utilized. The results show that for 
large planning problems, the hierarchical approach requires significantly less computational effort than the monolithic approach. However, the sub-optimality of the solution obtained by the hierarchical approach increases as the problem size increases.

The proposed hierarchical scheme permits the computation of aggregate as well as detailed production plans when detailed demand/forecast information is not known (or not considered necessary) at high management levels with long planning horizons. It also allows absorption of random events without frequent recomputation. For faster computations parallel algorithms could be employed. This approach can be particularly useful when different criteria exist at different levels of the hierarchy. A useful extension would be to consider multiple routings for part types inside each cell and to include setup costs.

\section{Acknowledgment}

This work is supported in part by the Institute for Systems Research of the University of Maryland under grant \# NSFD CD 8803012.

\section{References}

[1] A.C. HAX, H.C. MEAL, "Hierarchical Integration of Production Planning and Scheduling," In Studies in the Management Sciences (M.A. Geisler, Ed.), North Holland - American Elsevier, pp. 53$79,1975$.

[2] J. HERRMANN, A. MEHRA, I.MINIS, J.M. PROTH, "Hierarchical Production Planning with Part, Spatial and Time Aggregation," Technical Report, Institute for Systems Research, University of Maryland, College Park, MD 20742, 1994.

[3] A. MEHRA, I.MINIS, J.M. PROTH, "Hierarchical Production Planning for Complex Manufacturing Systems," accepted in 14th ASME International Computers and Exposition Conference, Minnesota, September, 1994.

[4] G. H. SAAD, "Hierarchical Production-Planning Systems: Extensions and Modifications," The Journal of the Operational Research Society, 41, pp. 609-624, 1990.

\section{Appendix}

In this appendix, we present the monolithic linear programming problem. The optimal solution of this
LP is the best solution that the hierarchical model presented in this paper can reach. The optimal criterion value of the LP presented below will be utilized to evaluate the effectiveness (sub-optimality) of the hierarchical scheme.

The problem is solved for the optimum production plan (variables $u_{j, w}^{k}$ ) and the optimum backlogging/holding costs (variables $P_{j, n_{j}}^{k}$ ). The production planning problem can be formally stated as follows:

$$
\begin{gathered}
\operatorname{minimize} \sum_{k \in H} \sum_{j \in \mathcal{P}}\left\{\left(\sum_{w=1}^{n_{j}-1} I_{j, w}\right.\right. \\
\left.\left(s_{j, w}^{0}+\sum_{a=1}^{k}\left(u_{j, w}^{a}-u_{j, w+1}^{a}\right)\right)+P_{j, n_{j}}^{k}\right\} \\
\text { subject to: } \\
P_{j, n_{j}}^{k} \geq I_{j, n_{j}} \cdot\left(s_{j, n_{j}}^{0}+\sum_{a=1}^{k}\left(u_{j, n_{j}}^{a}-d_{j}^{a}\right)\right) \\
P_{j, n_{j}}^{k} \geq-B_{j} \cdot\left(s_{j, n_{j}}^{0}+\sum_{a=1}^{k}\left(u_{j, n_{j}}^{a}-d_{j}^{a}\right)\right) \\
\sum_{j \in \mathcal{P}} \sum_{w \in \mathcal{K}_{j}} \delta_{j, w}(i) \cdot u_{j, w}^{k} \cdot t_{j, w} \leq T ; \forall i \in M \\
\sum_{a=1}^{k} \leq s_{j, w-1}^{0}+\sum_{a=1}^{k-1}\left(u_{j, w-1}^{a}-u_{j, w}^{a}\right) ; \forall w \in \mathcal{R}_{j} \\
\sum_{k \in h(\kappa)} \sum_{j \in f} u_{j, v_{j}^{q}-1+1}^{k} \leq \sum_{j \in f}(10) \\
\left.\left(u_{j, v_{j}^{q}-1}^{a}-u_{j, v_{j}^{q-1}+1}^{a}\right)\right) ; \forall f \in \mathcal{F}, q \in \widehat{\mathcal{R}}_{f}, \kappa \in H A \\
u_{j, w}^{k} \geq 0 ; \forall w \in \mathcal{R}_{j} .
\end{gathered}
$$

$\forall j \in \mathcal{P}, k \in H$ in constraints $(11-13,15,17)$, and $\forall k \in H$ in constraint (14)

The objective function (10) consists of both (i) the inventory holding costs of the work-in-process (for $\left.w=1, \ldots, n_{j}-1\right)$ and the finished part types $\left(w=n_{j}\right)$ and (ii) the backlogging costs of the finished part types over the entire planning horizon. Constraints (11) (13) represent these costs. The capacity constraints on the work-centers for all the elementary periods of the horizon are represented by (14). Constraint (15) denotes that the total instances of an operation on a certain part type within an elementary period cannot exceed the number of part types contained in the upstream buffer at the end of the previous elementary period (assumption A3). Constraint (16) satisfies assumption A4. Finally, (17) represents the nonnegativity of production. 